\title{
Signatures of pulsations and mass loss in the spectra of post-AGB stars
}

\author{
Laimons Začs ${ }^{1}$, Julius Sperauskas ${ }^{2}$, Aija Laure ${ }^{1}$ and Olesja Smirnova ${ }^{1}$ \\ ${ }^{1}$ Faculty of Physics and Mathematics, University of Latvia, Raiņa bulvāris 19, LV-1586 Rīga, \\ Latvia \\ email: zacs@latnet.lv \\ ${ }^{2}$ Vilnius University Observatory, Ciurlionio 29, Vilnius 2009, Lithuania \\ email: julius.sperauskas@ff.vu.lt
}

\begin{abstract}
The results of high-resolution spectroscopy and radial velocity monitoringa are presented for selected post-AGB candidate stars. Time series of high-resolution spectra for HD235858 shows spliting of low-excitation atomic lines and significant changes in the intensity of $C_{2}$ and $\mathrm{CN}$ lines originated in the extended atmosphere significantly affected by pulsations and outflow. Mass ejection was confirmed for cool R CrB candidate stars DY Per, V1983 Cyg, and V2074 Cyg.
\end{abstract}

Keywords. Stars: AGB and post-AGB, stars: oscillations, stars: winds, outflows

\section{Observations and analysis}

Radial-velocity of HD 235858 was monitored using the CORAVEL spectrometer (Upgren et al. 2002) of the Vilnius University installed on the $1.65 \mathrm{~m}$ telescope at the Moletai Observatory (Lithuania) and on the $1.6 \mathrm{~m}$ and $2.3 \mathrm{~m}$ telescopes at the Steward Observatory (Arizona) with the standard deviation of one measurement of about $0.4-0.7 \mathrm{~km} \mathrm{~s}^{-1}$. High-resolution spectra for HD 235858 and DY Per were obtained with the coudé échelle spectrometer MAESTRO $(R \sim 45,000)$ on the $2 \mathrm{~m}$ telescope at the Observatory on the Terskol Peak in Northern Caucasus (altitude of $3100 \mathrm{~m}$ ) equipped with a Wright Instruments CCD detector. High resolution spectra for V1983 Cyg, V2074 Cyg, and DY Per were observed on August 20-24th 2008 with the FIbre-fed Échelle Spectrograph (FIES) installed at the $2.5 \mathrm{~m}$ Nordic Optical Telescope (NOT) on La Palma with a resolving power of 67,000 . All spectra were bias subtracted, flat field corrected, and converted to one-dimensional spectra using the standard DECH20T package. The search for periods was carried out using Fourier analysis software Period04 (Lenz \& Breger 2005).

\section{Results and conclusions}

Radial velocity monitoring of HD 235858 with the CORAVEL spectrometer revealed variations with a period of about 130 days and a peak-to-peak amplitude of about $10 \mathrm{~km} \mathrm{~s}^{-1}$. The radial velocity curve displays obvious variations, therefore, secondary period was suspected (Začs et al. 2009).

A number of narrow circumstellar envelope (CSE) lines of $C_{2}(3,0),(2,0)$ Phillips system and $\mathrm{CN}(2,0)$ Red system are identified. The expansion velocity of CSE was measured, $V_{\exp }$ $=-8.4 \pm 0.5 \mathrm{~km} \mathrm{~s}^{-1}$, in agreement with the value measured using $\mathrm{CO}$ lines, $V_{\exp }(\mathrm{CO})=$ $-8.5 \mathrm{~km} \mathrm{~s}^{-1}$ (Hrivnak \& Bieging 2005). 

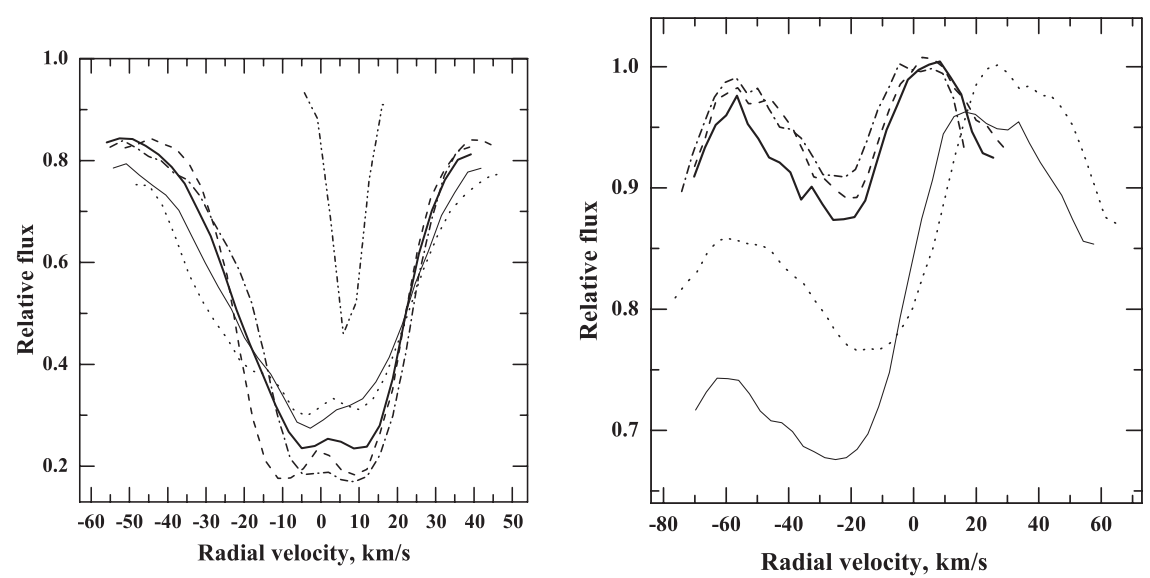

Figure 1. The profiles of Ba II line at $6496.93 \AA$ (left panel) and $C_{2}(0,1)$ bandhead at $5635 \AA$ (right panel) in the radial velocity scale relative to the systemic velocity of $-40.1 \mathrm{~km} \mathrm{~s}^{-1}$ in the spectra of HD 235858 observed on November 2002 (thick solid), October 2006 (thin solid), November 2006 (dotted line), February 2008 (dashed line), and September 2010 (dotted-dashed line). Two/three components and extended blue wings are observed in strong low excitation lines. Telluric line is shown to illustrate resolution. The bandhead of $C_{2}$ is variable and blueshifted relative to the photospheric atomic lines by about $-20 \mathrm{~km} \mathrm{~s}^{-1}$.

Strong low-excitation atomic lines in the spectra of HD 235858 are variable and disturbed by splitting (see Fig. 1, left panel). The movement of layers in pulsating extended atmospheres influenced by shock waves could be the physical mechanism of splitting.

Variable molecular features are observed in the spectra of HD 235858. Molecular lines are enhanced in comparison with the standard photosphere of G5 supergiant and a significant variability in the positions and intensities was found (see Fig. 1, right panel). Variable molecular features are formed apparently in the cool outflow of HD 235858.

DY Per seems to be a prototype of Galactic cool R CrB star with confirmed hydrogen deficiency, high carbon abundance, high ${ }^{12} \mathrm{C} /{ }^{13} \mathrm{C}$ ratio, solar metallicity, and confirmed episodes of mass ejections (Začs et al. 2007).

The evolutionary status of V1983 Cyg and V2074 Cyg is not clear. Episodes of mass ejection are confirmed using Na I D1 and D2 lines. However, these cool carbon stars with solar metallicity and significant enhancement of neutron-capture elements are rather luminous AGB stars like W CMa and U Hya.

\section{References}

Hrivnak, B. J. \& Bieging, J. H. 2005, ApJ, 624, 331

Lenz, P. \& Breger, M. 2005, CoAst, 146, 53

Upgren, A. R., Sperauskas, J., \& Boyle, R. 2002, Baltic Astron., 11, 91

Začs, L., Mondal, S., Chen, W. P., Pugach, A. F., Musaev, F. A., \& Alksnis, O. 2007, A\&A, 472,247

Začs, L., Sperauskas, J., Musaev, F. A., Smirnova, O., Yang, T. C., Chen, W. P., \& Schmidt, M. 2009, ApJ, 695, L203 